\section{Josef Roos}

Die Aeskulap-Klinik in Brunnen ist das Schweizer Kompetenzzentrum für ärztliche Ganzheitsmedizin. In stimmungsvoller Hotelatmosphäre und schönster Naturlandschaft am Vierwaldstättersee erhalten die stationären wie auch ambulanten Patienten qualifizierte, integrative schul- und komplementärmedizinische Behandlung.

\section{Interdisziplinäre Vorgehensweise}

Die Aeskulap-Klinik bietet ihren Patienten alle Voraussetzungen für eine erfolgreiche Behandlung und nachhaltige Genesung. Die medizinische Qualifikation und Erfahrung des interdisziplinären Ärzteteams, das Engagement der geschulten Mitarbeiter, die Zimmer mit Blick auf die Berge und die Vollwertkost versteht die Klinik als Beitrag für alle Patienten, in familiärer Klinikatmosphäre gesundheitliche Probleme zu kurieren.

Fast alle der 25 Ärzte und Zahnärzte sind Fachärzte diverser Disziplinen mit komplementärmedizinischer Zusatzausbildung und Erfahrung. Im Sinne eines individuellen Ansatzes geben sie beiden Fachbereichen heilsamen Platz. So wird das Beste aus

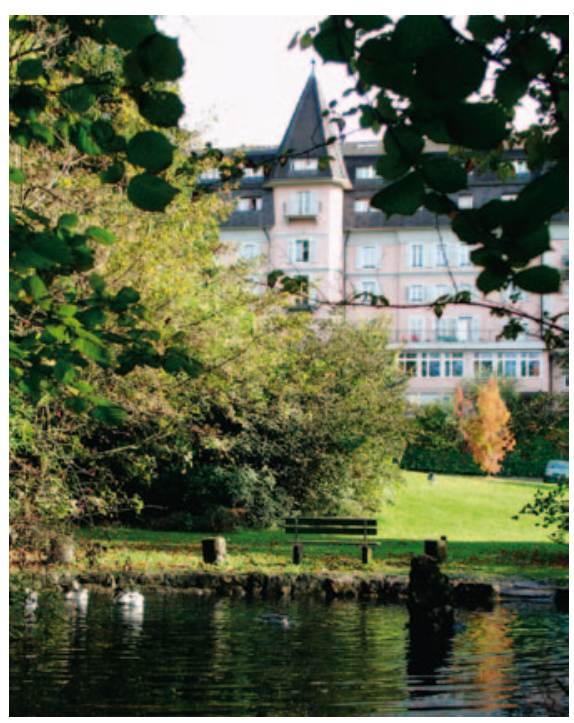

\title{
20 Jahre Aeskulap-Klinik in Brunnen
}

\section{Das Kompetenzzentrum für ärztliche Ganzheitsmedizin}

Seit 1990 behandelt die Aeskulap-Klinik ihre Patienten im Sinne der Integration von Schul- und Komplementärmedizin. Wie kaum anderswo werden beide Elemente harmonisch zum Wohle der Patienten eingesetzt - individuell und mit viel Zeit. Im Aeskulap-Team arbeiten 25 Ärzte und Zahnärzte, die sich vor allem auf integrative Onkologie, Schmerzbehandlung, psychosomatische Leiden sowie Orale Medizin konzentrieren. Die Aeskulap-Klinik - das Krankenhaus mit viel Herz und Wissen - feiert 2010 ihren 20. Geburtstag.

Schul- und Komplementärmedizin zu einem integrativen und individuellen Ansatz zusammengeführt.

Für körperliche und psychische Probleme Die Aeskulap-Klinik ist Ansprechpartner bei primär langjährigen, komplexen, körperlichen und psychischen Problemen und ist für Menschen jeden Alters und jeder Versicherungskategorie da. Dabei fokussiert sich die Klinik hauptsächlich auf integrative Krebsbehandlung, Schmerztherapie, chronische Leiden (unter anderem chronische Infektionen, Allergien, gastrointestinale Erkrankungen, Hautprobleme), psychosomatische Erkrankungen (z.B. Depressionen oder Burnout) und Orale Medizin (integrative Zahnmedizin).

Interdisziplinäre, individuelle Behandlung wird in der Aeskulap-Klinik gross geschrieben. Bei ihr nehmen sich die Ärzte und Therapeuten noch Zeit für den Menschen. Der Wert und Kern der Medizin liegt nicht nur im Heilen, sondern vor allem darin, dass sich jemand eines anderen Menschen in seiner Not annimmt.

\section{Der Mensch in seiner Gesamtheit steht im Mittelpunkt}

Die Ärzte der Aeskulap-Klinik treten mit einer ganzheitlichen Betrachtungsweise an ihre Patienten heran, in welcher die Komplementärmedizin eine entscheidende Rolle spielt. Nicht die Symptome einer Krankheit stehen
Aeskulap-Klinik - das integrative Kompetenzzentrum für

- Krebserkrankungen (integrative Onkologie);

- chronische Schmerzen;

-psychosomatische Krankheiten, z.B. Burnout und Depression;

- chronische Leiden wie unter anderem Rheuma, Hautkrankheiten, gastrointestinale Probleme, Infektionen, Allergien; -Zahn-, Mund- und Kiefererkrankungen (Orale Medizin).

im Mittelpunkt, sondern der Mensch in seiner Gesamtheit und im komplexen Zusammenspiel von Körper, Seele und Geist. Eine Heilung kann nur durch die Stimulation der Selbstheilkräfte und die Erlangung der gesundheitlichen Balance erreicht werden. Eine Reihe von regulativen Massnahmen, wie z.B. Wärmeanwendungen, Sauerstofftherapie, klassische Homöopathie oder Neuraltherapie, stehen den Patienten zur Verfügung.

Die Schulmedizin hat ihre grossen Verdienste. Und es gilt, sie dort einzusetzen, wo sie ihren grössten Nutzen hat, wie z.B. in der Chirurgie, in der Notfallmedizin oder bei Unfällen. Wo sie Chronifizierung und Nebenwirkungen erzeugt, ist sie durch komplementäre Verfahren zu ergänzen, allenfalls auch zu ersetzen. In diesem Sinne sind Schul- und Komplementärmedizin zu einer integralen Ganzheitsmedizin zu verbinden. Dieser ganzheitlichen Sichtweise hat sich die Aeskulap-Klinik verschrieben. (c) 2010 S. Karger GmbH, Freiburg
Dr. Josef Roos

Aeskulap-Klinik

Ärztliche Ganzheitsmedizin

Gersauerstrasse 8, 6440 Brunnen am Vierwaldstättersee, Schweiz

Tel. +41 41 82549-49, Fax -65

info@aeskulap.com 